\title{
Virtual Reality Demonstration of Surgical Specimens, Including Links to Histologic Features
}

\author{
Hector Melin-Aldana, M.D., Debra Sciortino \\ Children's Memorial Hospital, Northwestern University Feinberg School of Medicine, Chicago, Illinois
}

The demonstration of surgical specimens, whether using 35-mm slides or digital images, tends to consist of the sequential presentation of images. Current digital technology permits a more flexible and effective way of communication, with the opportunity to more easily "navigate" between different aspects of specimens. We demonstrate a "virtual reality" method, based on QuickTime VR technology, that permits the interactive review of a complete profile of surgical specimens in the horizontal plane. Specimens were placed individually on a circular rotating platform. Thirty-six images of each specimen were captured using a digital camera, with rotation of the platform at $10^{\circ}$ intervals. The images were transferred to a computer and processed using specialized software (VRWorx). Histologic images were separately captured from tissue sections on glass slides using a digital camera mounted on a microscope. The final product is viewed using the QuickTime Viewer software application. A $360^{\circ}$ horizontal view of the specimens is achieved, with the capacity to actively rotate the specimen and to zoom in for closer review. Additionally, the user/presenter can click in predetermined "hot spots," which will open histologic images linked to those spots. This methodology, which uses readily available computer technology, helps provide a better three-dimensional understanding of surgical specimens and also a better correlation between gross and microscopic features.

KEY WORDS: Computers, Digital imaging, Surgical pathology, Virtual reality, VR.

Mod Pathol 2003;16(9):958-961

Copyright ( $(2003$ by The United States and Canadian Academy of Pathology, Inc.

VOL. 16, NO. 9, P. 958, 2003 Printed in the U.S.A

Date of acceptance: May 13, 2003.

Presented at the 91st Annual Meeting of the US and Canadian Academy of Pathology, February 23-March 1, 2002, Chicago, Illinois. Published in abstract form in Modern Pathology 2002:15:342A.

Address reprint requests to: Héctor Melín-Aldana, M.D., Department of Pathology, Children's Memorial Hospital, 2300 Children's Plaza, Box 17, Chicago, IL 60614; fax: 773-880-8127; e-mail: h-melin@northwestern.edu.

DOI: 10.1097/01.MP.0000085597.48271.BD
Recent advances in photographic and computer technology allow pathologists to easily capture digital images of surgical specimens. Because of these advances, digital technology has greatly improved the process of manipulation, presentation, distribution, and archiving of images. However, the digital presentation of surgical specimens still tends to be performed in a two-dimensional manner, not taking full advantage of this new technology.

Virtual reality (VR) technology, also described as immersive imaging, provides the opportunity to present, and interact with, specimens as threedimensional structures $(1,2)$. This approach permits a better understanding of the features of the specimens by allowing the demonstration of structural details from many angles. Previously, the applications of VR technology to the study of anatomic structures had required developers to use complex methodology (35). Moreover, the end user was sometimes required to use special and/or cumbersome equipment, such as a head-mounted crystal display, or goggles and a projection system (5). QuickTime VR (QTVR) technology, introduced in 1996 by Apple Computer (Cupertino, CA; www.apple.com), permits the creation of VR images, called object movies, using standard, easily available, and affordable equipment and easy-to-use methodology $(1,6)$. With the use of QTVR technology, movies of surgical specimens can be rotated and observed from multiple angles, including zooming in and out. Links to diagrams or to images of microscopic features from specific areas of the specimens can be easily added. QTVR movie files can be distributed by electronic mail, CD-ROM or DVD, or by incorporation in World Wide Web sites. The movies can then be viewed using standard computer equipment. This methodology has been proven easy to use and successful in the teaching of anatomic structures $(1,2$, 5). In this article we describe our use of QTVR technology in the demonstration of surgical specimens.

\section{MATERIALS AND METHODS}

For the acquisition of images to be used for the creation of QTVR movies, we slightly modified the area already used in our department for photo- 
graphing surgical specimens. In QTVR methodology, the camera is placed in a horizontal plane with respect to the specimen (see more detailed description below), whereas for regular photography, the camera is usually mounted above the specimen. First, the surgical specimen is placed in a rotating platform. Specimens that are not sufficiently firm to maintain a constant position in the platform are fixed overnight in $10 \%$ buffered formalin. If representative tissue sections need to be taken before fixation in formalin, this is done carefully, trying not to alter the specimen's macroscopic features. We use an inexpensive circular plastic platform (commonly called a "lazy Susan" in the United States), in which we have placed marks every $10^{\circ}$. The specimen is inserted in a vertically oriented metal rod which is affixed to the center of the rotating platform. We sometimes also use modeling clay to provide additional support. Our setup is similar to that described by Trelease et al. (1). Lighting of the specimen is provided by flood lamps placed at $45^{\circ}$ angles on both sides of the specimen, and we use a blue plastic board as background. The lamps are those already set up for regular photography of specimens, as is the blue plastic board. To obtain a movie that will be rotated completely $\left(360^{\circ}\right)$ in the horizontal plane, 36 images are taken, with manual rotation of the platform and capture of one image at every $10^{\circ}$ mark. We use a Kodak DC260 digital camera placed on a tripod, and the same exposure and lighting conditions are maintained throughout all images. The images are captured in high-quality JPEG format, stored in a flash card located inside the camera, and transferred via a flash card adaptor to a Macintosh G3 PowerBook.

Once in the computer's hard drive, each image is individually improved with respect to size, brightness, contrast, and elimination of artifacts, using Adobe Photoshop 7.0 (Adobe Systems Incorporated, San Jose, CA; www.adobe.com). Creation of the final QTVR object movie is done using The VR Worx, Version 2.1 (VR Toolbox, Inc., Pittsburgh, PA; www.vrtoolbox.com). The creation of the movie is a semiautomated process involving the following steps: (1) initial setup, in which the size (in our case, $800 \times 600$ pixels), number (36) and spacing $\left(10^{\circ}\right)$ of images are indicated; (2) addition of links to microscopic images, known as hot spots; (3) compression, if desired (we use JPEG compression with maximum quality settings); and (4) export of the final movie file.

The final movies are in QuickTime (*.mov) format, and the QuickTime Player is used to view them. This free application is already installed in many computers or can be easily downloaded from Apple Computer's Website (www.apple.com/quicktime). Macintosh and Windows versions are available. To incorporate the movies into a World Wide
Web site, specific HTML code is used $(1,7,8)$. The files can be viewed in any graphics-based Internet browser (Safari, Netscape, Internet Explorer, Mozilla, Opera, etc.), using the QuickTime plug-in. Again, this plug-in is also already installed in many computers or can be downloaded free of charge from Apple Computer's Website (see above).

\section{RESULTS}

The reader is encouraged to review the examples at www.hmelin.net/qtvr.html to fully appreciate the features and potential of QTVR technology. In the first example, a liver showing macronodular cirrhosis from a patient with cystic fibrosis is demonstrated (Fig. 1). This specimen was obtained when the patient underwent liver transplantation. Rotation of the specimen is accomplished by either of two methods. In the first method, when the computer cursor has turned into a hand icon, the computer mouse button is clicked and held, and the liver is rotated by dragging the cursor horizontally (Fig. 1). The second method is by placing the cursor at any of the horizontal edges of the image (Fig. 1). When the cursor has turned into a horizontal arrow, the liver is rotated by clicking the mouse button. The buttons at the bottom of the QuickTime Player window that are used in QTVR movies are as follows (Fig. 1, left to right): volume (if the movie includes sound), return to the previous image, zoom out, zoom in, reveal hot spots, and a link to Apple Computer's QuickTime Website, if the computer is connected to the Internet. This particular

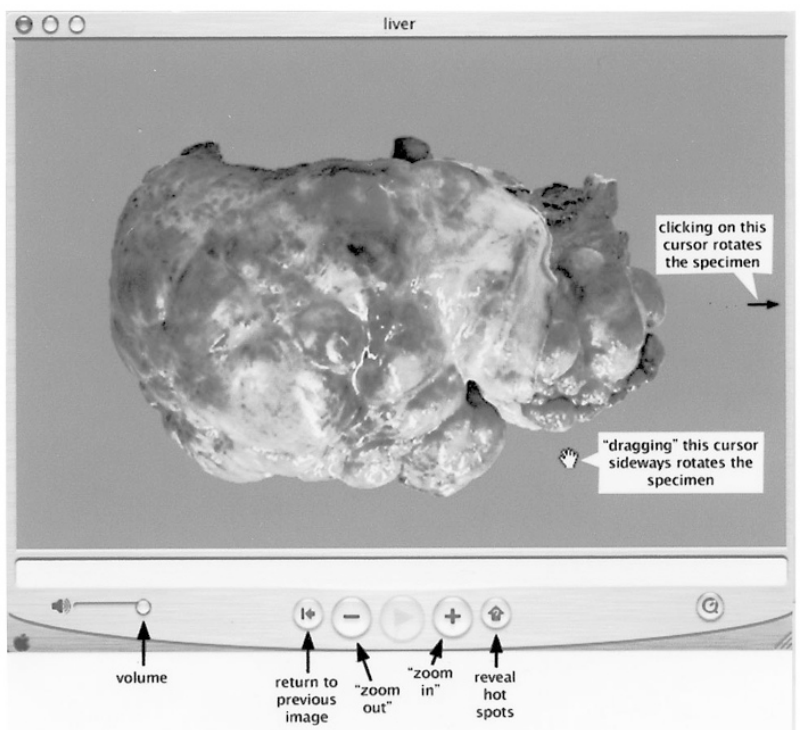

FIGURE 1. Window of the QuickTime Player showing a QTVR movie of a liver with macronodular cirrhosis. The arrows and boxes describe the functions of the different cursors and buttons that are applicable to QTVR movie files. The "return to previous image" button applies to the use of hot spots. See Figures 2 and 3 for examples of hot spots. 
example does not include links (hot spots) to microscopic images because the focus is on the prominent macroscopic features of the specimen.

In the second example, a pulmonary lobe from a patient with cystic fibrosis (not the same patient) is demonstrated (Fig. 2). As can be appreciated, the pleural surface showed no specific lesions. The cut surface includes bronchi containing mucus plugs, dilated blood vessels, and grossly unremarkable pulmonary parenchyma. As shown, the hot spots are revealed by clicking on the appropriate button of the QuickTime Player. Clicking on the hot spots opens images of the microscopic features of those specific areas (Fig. 3). The return to the original movie of the specimen is accomplished by clicking on the appropriate button of the Player.

\section{DISCUSSION}

We have described the use of QTVR technology for the demonstration of surgical specimens, a technology that we use in our practice of pediatric surgical pathology. We believe that this interactive technology permits a better understanding of the three-dimensional nature of specimens.

From the point of view of the developer, QTVR technology has the advantage of not requiring the use of very expensive, complicated hardware or software. Pathology departments that are already set up for photographing surgical specimens, whether the setup is for analog or digital photography, will not require a significant investment for also producing QTVR movies. The equipment that we routinely use is easily available. Our digital camera is a consumer-level camera. We use a Macintosh PowerBook G3 laptop computer, which achieves very satisfactory production time and results. This computer was already in use in our department, it was not specifically purchased for the creation of QTVR movies, and it is not specifically dedicated to this purpose. For preparation of the

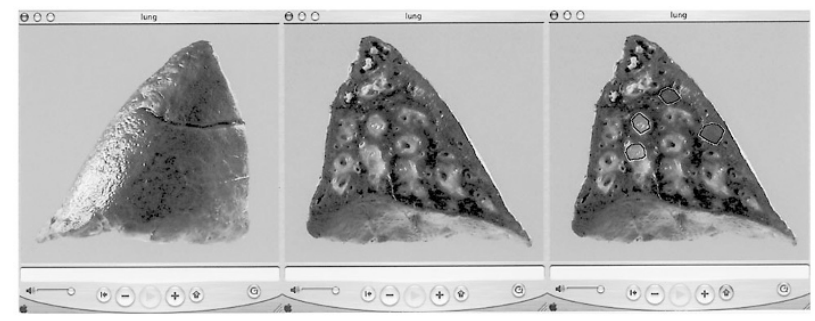

FIGURE 2. Composite figure including three windows of the QuickTime Player showing a movie of a pulmonary lobe removed from a patient with cystic fibrosis. The image on the left corresponds to the initial view, the pleural surface. The image in the center shows the cut surface, reached after rotation of the specimen. The image on the right reveals the assigned hot spots, accomplished by clicking the corresponding button at the bottom of the Player (see Fig. 1 for description of the buttons).

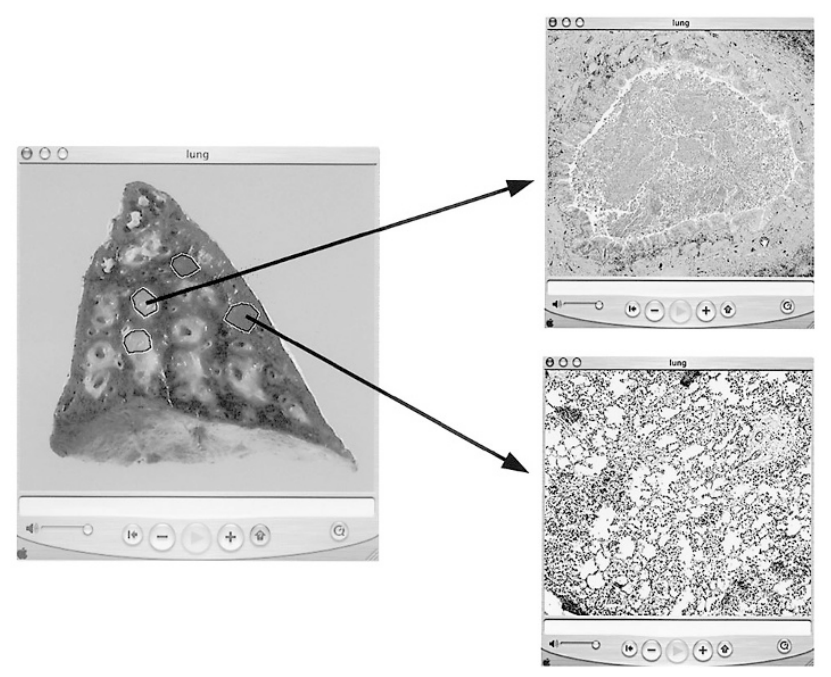

FIGURE 3. Diagrammatic representation of the links of the "hot spots" in the movie of the pulmonary lobe. Clicking on each hot spot opens an image of the histologic appearance of that area. The microscopic image is opened in the same window of the QTVR Player, replacing the original movie. To return to the movie, the left button at the bottom of the Viewer is clicked (see Fig. 1 for description of the buttons).

images before creation of the movies, we use Adobe Photoshop (Adobe Systems Inc.), which is a professional-level image editing software with complexity and price that might be intimidating to some pathologists. However, many simpler and less expensive image editing programs are available. For production of our QTVR movies, we use The VR Worx (VR Toolbox, Inc.), which has Macintosh and Windows versions. We are aware of only few other software tools to create QTVR object movies, such as Make QTVR Object (free, Apple Computer Inc.), and QuickTime Authoring Studio (Apple Computer Inc), but we have not used them and therefore are not familiar with their features. The incorporation of QTVR movies into World Wide Web pages can be achieved with standard HTML coding and by using software already included in the Macintosh (SimpleText, TextEdit) or Windows (Notepad) operating systems. From the point of view of the user, currently available computer hardware is adequate, and the required software (QuickTime Player, see Methods section, above) is easily available and free. Our movies can be rotated in a horizontal plane, but object movies that rotate in multiple planes can be created. However, this significantly adds time, equipment, expense, and complexity to the process (6). We have not created multiple-plane QTVR movies yet.

QTVR object movies, like any other type of image, are invaluable when surgical specimens are no longer intact, having been significantly altered by the necessary manipulation of regular processing. Every pathologist has witnessed the surgeon's frustration when she or he wants to review the speci- 
men but is unable to reconstruct it after it has been inked, cut, and sampled; the availability of an interactive, three-dimensional image of the specimen in its original state is very helpful. QTVR movies are also of great potential use in teaching, when it is not possible or practical to maintain an adequate organ "museum," such as a collection of hearts with congenital anomalies. The latter application is particularly relevant in light of the recent controversy surrounding organ retention by pathologists (9).

QTVR movies can be easily distributed, via CDROM, electronic mail, or incorporation into World Wide Web sites. However, to obtain optimal image quality, one has to be aware that the size of these files tends to be large. Because our main use of these movies is the presentation of surgical specimens at conferences, projected directly from a computer, we create our QTVR movies at $800 \times 600$ pixels, at high resolution, and with light compression, and the resulting files are between 4 and 10 megabytes in size, depending on the number of linked histologic images. However, the size of the files may be significantly decreased by decreasing image size and resolution and by using a higher compression setting. These measures will have a detrimental effect on the quality of the final movie, and a compromise must be reached between movie quality and ease of access. These issues are particularly relevant when incorporating QTVR movies into World Wide Web sites, because the interactive experience will be negatively affected if the user has to wait a long time to view the movies.

We have encountered few disadvantages of incorporating QTVR technology into our practice. Some specimens are not suitable for placement in the rotating platform, particularly when they are too soft, too small (or too big), or have awkward shapes. Sometimes the best macroscopic features of a specimen are lost because extensive sampling must be performed before formalin fixation. Taking $36 \mathrm{im}-$ ages manually is time-consuming for the pathologist, and therefore the use of this technology should be reserved for specific specimens, or the participation of a technician (pathology assistant) should be established. Image acquisition can be automated by using a camera connected to a dedicated computer that automatically controls the rotation of the platform and captures the images. These setups are commercially available (6), but their prices are probably beyond the budgets of most pathology departments. We do not have such equipment and thus cannot comment on its efficacy. Improving each digital image, such as by manipulating brightness and contrast and eliminating artifacts, can be time-consuming, but this step can be easily and significantly shortened or even eliminated by careful setup of the specimen during ac- quisition. Again, the participation of a technician in this step, and not the pathologist, is an option. Making the final movie does not generally involve a significant length of time, because it involves only selecting the appropriate software settings and incorporating the hot spots.

It has been demonstrated that QTVR technology is an effective teaching tool. Nieder et al. (2) conducted a study in which this technology was used to teach the anatomy of the skull. Their study group consisted of 90 1st-year medical students. Feedback was solicited from the students as part of the course evaluation, and a majority indicated that the program was easy to use and provided appropriate information. In another study, the subjects reported more efficient learning of object structure using this interactive technology, compared with using passive observation (5). Informal feedback provided to us after the use of QTVR technology to demonstrate specimens has been consistently positive.

In summary, we propose that the use of QTVR technology has an important place in the demonstration of surgical specimens, making a significant contribution to the teaching of anatomic pathology.

Acknowledgments: We are indebted to Francisco Gonzalez-Crussi, M.D., for his valuable contributions during review of this manuscript.

\section{REFERENCES}

1. Trelease RB, Nieder GL, Dorup J, Hansen MS. Going virtual with QuickTime VR: new methods and standardized tools for interactive dynamic visualization of anatomical structures. Anat Rec (New Anat) 2000;261:64-77.

2. Nieder GL, Scott JN, Anderson MD. Using QuickTime virtual reality objects in computer-assisted instruction of gross anatomy: Yorick-the VR skull. Clin Anat 2000;13:287-93.

3. Hoffman HM, Murray M, Irwin AE, McCracken T. Developing a virtual reality-multimedia system for anatomy training. Stud Health Technol Inform 1996;29:204-10.

4. Kling-Petersen T, Pascher R, Rydmark M. Virtual reality on the web: the potentials of different methodologies and visualization techniques for scientific research and medical education. Stud Health Technol Inform 1999;62:181-6.

5. James KH, Humphrey GK, Vilis T, Corrie B, Baddour R, Goodale MA. "Active" and "passive" learning of threedimensional object structure within an immersive virtual reality environment. Behav Res Methods Instrum Comput 2002; 34:383-90.

6. Kitchens SA. Capturing images for object movies. In: Kitchens SA, editor. The QuickTime VR book. Berkeley, CA: Peachpit Press; 1998. p. 91-101.

7. Kitchens SA. Delivery to the web. In: Kitchens SA, editor. The QuickTime VR Book. Berkeley, CA: Peachpit Press; 1998. p. 161-88.

8. Putting QuickTime in a web page. Quick Time for the web. San Diego, CA: Academic Press; 2002. p. 29-45.

9. Bradbury J, Weber W. Consent requirements for necropsy may change in UK. Lancet 1999;354:2055. 